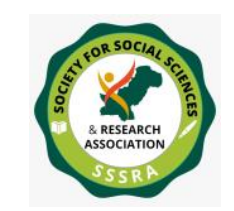

Psychological Comorbidity of Breast Cancer ...

\title{
Psychological Comorbidity of Breast Cancer: A Cross-Sectional Study from Pakistan
}

\author{
Tayyeba Kiran \\ Phd Fellow- Department of Psychology \\ University of Karachi \\ Karachi - Pakistan \\ tayyebakiran@gmail.com \\ Prof. Dr. Anila Amber Malik \\ Professor of Psychology \\ Department of Psychology \\ University of Karachi \\ Anilaahsan@hotmail.com
}

\begin{abstract}
:
Background: Psychological comorbidity of breast cancer is a serious global health problem that inflicts huge economic and societal costs to patients themselves, families, as well as healthcare systems. The aim this study was to estimate the prevalence of common mental disorders (anxiety and depression) in breast cancer patients.
\end{abstract}

Methods: The present study is cross sectional study that was a secondary analysis of a Randomized Controlled Trial in Pakistan. A total of 402 breast cancer patients were recruited from both public and private hospitals in Karachi Pakistan. Patients were assessed for depression using the structured questionnaire "Patient Health Questionnaire (PHQ-9)" and anxiety using the "Generalized Anxiety Disorder scale (GAD-7)".

Findings: Participants' mean age was 38.9 years, majority had no formal education (77.4\%). Their mean duration of breast cancer illness was 28.9 months. A large percentage of the study sample was found to be anxious (59.7\%), depressed (69.4\%) and had suicidal ideation (47.5\%).

Conclusion: High prevalence of anxiety, depression and suicidal ideation in breast cancer patients highlights the vital role of carrying out regular 


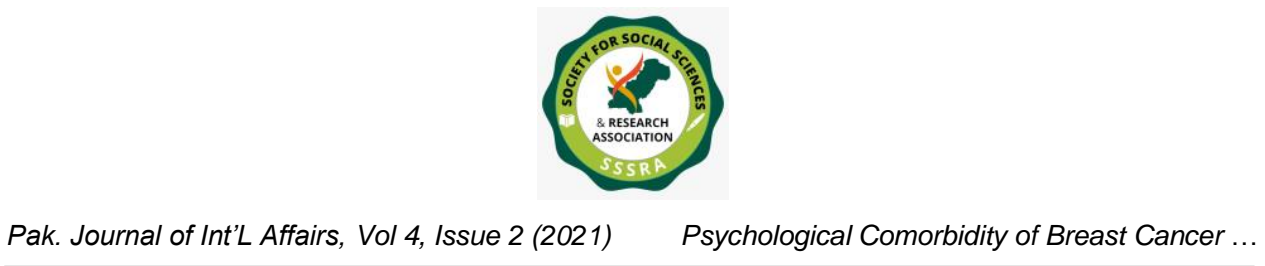

psychosocial assessments and offering the necessary, culturally relevant support to these patients.

Keywords: Breast cancer; Depression; Anxiety; Suicidal ideation; Pakistan

\section{Introduction}

Breast cancer is the most commonly occurring cancer among females globally, effecting approximately 2.1 million females annually (WHO, 2018). Breast cancer is the greatest contributor to cancer-related mortality among females across the world. It was reported that in 2018 a total of 627,000 deaths occurred because of breast cancer which is approximately $15 \%$ of all deaths caused by cancer in females (WHO, 2018). Mortality rates (annual) were reported to be much higher in South Asia (3.06\%) compared to high income countries such as the United States (1.69\%) (Ranganathan et al., 2018). In the time period between 2005 and 2015, mortality rates in South Asia has increased to approximately $27.9 \%$ (Ranganathan et al., 2018). Pakistan is amongst the Asian countries with one of the highest incidence rates of breast cancer (Menhas \& Umer, 2015). A research center in Pakistan reported that breast cancer is one of the frequently reported cancer $(45.4 \%)$ among women in Pakistan with young women being more vulnerable (Basra, Saher, Athar, \& Raza, 2016).

Common mental disorders (such as depression and anxiety) in breast cancer patients are associated with increased risk of cancer incidence and increased risk of mortality with their co-occurrence further increasing the risk of cancer mortality and posing a huge burden on healthcare systems (Shang, Hodge, Peng, He, \& Zhang, 2020). A systematic review (of 75 studies) also show that people with mixed physical and mental multimorbidity report greater risk of active safety incidents (such as adverse drug reactions) (Panagioti et al., 2015). Anxiety and/or depression are commonly reported experiences by breast cancer survivors even after many years of diagnosis and these experiences lead to greater disability and have adverse consequences for health-related quality of life (Rogers et al., 2017). In a recent systematic review of 72 studies, global estimates of depression in breast cancer patients were 32.2\% (Pilevarzadeh et al., 2019). A recent meta-analysis also reported high prevalence of anxiety (41.9\%) in this population (Hashemi et al., 2020).

Despite high prevalence of breast cancer in Pakistan, data on psychological comorbidities is limited and what is available is with a small sample size (Khan et al., 2016). Therefore, the present secondary analysis aimed at assessing prevalence of depression and anxiety in Karachi, the largest metropolitan city in Pakistan, and fifth largest city in the world and 


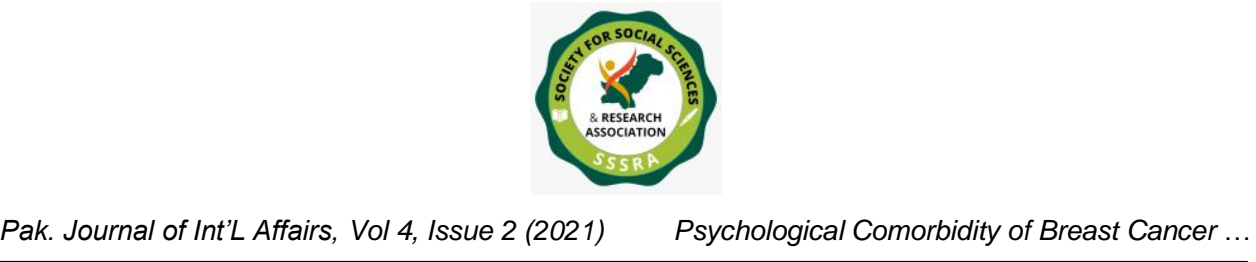

hence the findings may represent and highlight the needs of a large sized population. This study is secondary analysis from data collected as part of a multicenter Randomized Controlled Trial "Moving on After Breast Cancer" (trial registration number: NCT03571984; Ethics approval number: IPP/BU/OM103/1676)

\section{Material and Methods}

Setting: Patients were approached by a trained researcher in outpatient departments (OPDs) of oncology and medical units in two government run hospitals (Civil hospital, Abbasi Shaheed hospital) and a charity hospital (Dar-ul-sakoon) in Karachi.

Study design: Cross sectional research design

Sampling technique: A convenient sampling technique was used. Consecutive patients with breast cancer presented to participating hospitals were approached by a trained researcher and those who consented to participate were included in the study. These patients were originally screened for a Randomized Controlled Trial "Moving on After Breast Cancer" (NCT03571984).

Sample size calculation: A freely available Raosoft calculator (www.raosoft.com) was used for sample size calculation, considering $26 \%$ prevalence rate of depression in this patient population (Chen et al., 2010) with confidence interval of $95 \%$ and margin of error 5\%. The estimated minimum sample size was 292 .

Study duration: February 2018 - March 2020

\section{Instruments}

Socio-demographic question: A questionnaire was developed by the researcher to collect information about social and demographic variables such as; age, marital status, occupation, education, duration of $\mathrm{BC}$, and information about previous psychiatric illness.

Patient Health Questionnaire (PHQ-9) (Kroenke, Spitzer, \& Williams, 2001): The PHQ-9 is a structured, self -administered scale to assess level of depression. Eight items (out of nine) assess participants' depressive symptoms. The last question is about participants' functional impairment. Each question has 4 responses: "0-not at all", "1several days", "2-more than half the days", or "3-nearly every day". The overall score range is 0 to 27. Urdu translated PHQ-9 (Ahmad, Hussain, Akhtar, \& Shah, 2018) was 


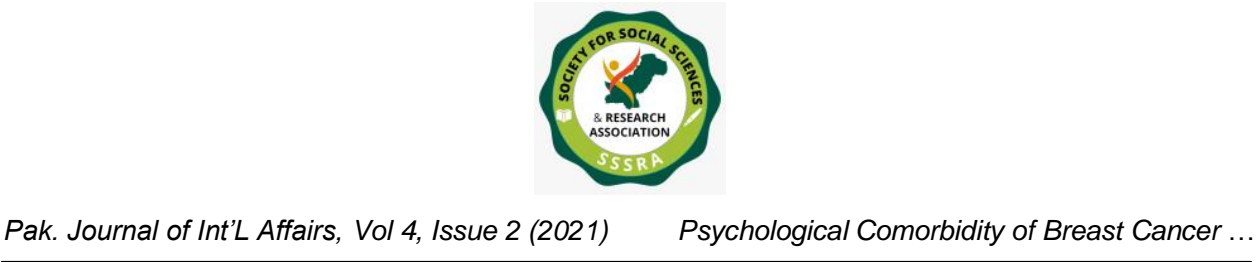

used in this study. The diagnostic validity of PHQ-9 was established in previously published researches ( 8 from primary care settings and seven studies conducted in clinics) and PHQ-9 score of $\geq 10$ had $88 \%$ sensitivity as well as specificity for depression (Maroufizadeh, Omani-Samani, Almasi-Hashiani, Amini, \& Sepidarkish, 2019).

Generalized Anxiety Disorder-7 (GAD-7) (Spitzer, Kroenke, \& Williams, 2006): Generalized anxiety disorder scale is a self-administered scale to assess symptoms of anxiety (Spitzer et al., 2006). It is comprised of seven items and each item has four responses, "0-not at all", "1-several days", "2-more than half the days", or "3-nearly every day". Total score ranges between 0-21. The Urdu version scale was used (Ahmad, Hussain, Shah, \& Akhtar, 2017) in this study. GAD-7 has shown to have good reliability and construct validity (Löwe et al., 2008).

\section{Procedure}

Ethics approval was sought for the main RCT from ethics review board (ERB) of Institute of Professional Psychology (IPP) (IPP/BU/OM/103/1676). The conduct of the research was in agreement with the Declaration of Helsinki. A trained researcher (clinical psychologist) approached breast cancer patients in participating hospitals. Detailed information about the study was provided to the treating physicians in recruiting OPDs who agreed to refer BC patients to the researcher. A total of 654 breast cancer patients were approached, out of which 402 were eligible and enrolled in the study. Out of 654 patients, for 82 patients it was difficult to answer questions because they were physically unwell, for 91 family of the patients did not give consent to the researcher to contact with the patients, for 47 family of the patient mentioned that patient has a serious mental disorder, and for rest of 32 patients treating consultants showed concern about patient's cognitive abilities that may impair their level of attention and their ability to concentrate. A detailed Participant Information Leaflet (PIL) was developed in Urdu and shared by the researcher with the patient and their carers and researcher also provided verbal information about the study. Written (or thumb impression) informed consent was taken from all the participants. Participants were provided with a copy of the signed informed consent one copy of the form was filed in Case Report Form (CRF) for researcher's record. The researcher asked the information about socio-demographic variables first and then administered PHQ-9 and GAD-7. All participants were reimbursed for their time.

\section{Statistics}

Statistical analysis was done using statistical software SPSS 23.0 for windows. Means and standard deviations for continuous data (such as age, duration of breast cancer, total 
anxiety scores, total depression scores etc) and frequencies and percentages for categorical data (such as marital status, severity categories of depression and anxiety, presence/absence of suicidal ideation) are presented.

\section{Results:}

Results show that majority of the women were adults between ages 25 - 60 years 346 (86.1\%), with no formal education 311 (77.4\%) and were housewives 366 (91\%). Their mean duration of breast cancer illness was 28.9 months (Table 1).

Table 1: Characteristics of breast cancer patients $(\mathrm{N}=402)$

\begin{tabular}{|c|c|c|}
\hline Characteristics & $\mathbf{n}$ & $(\%)$ \\
\hline \multicolumn{3}{|l|}{ Age (years) } \\
\hline Young adults $(18-25)$ & 43 & 10.7 \\
\hline Adults $(25-60)$ & 346 & 86.1 \\
\hline Older adults (61 and above) & 13 & 3.2 \\
\hline Mean (SD) & $38.90(11.86)$ & \\
\hline \multicolumn{3}{|l|}{ Education (\# of years of education) } \\
\hline No formal education & 311 & 77.4 \\
\hline Primary (1-5) & 22 & 5.5 \\
\hline Secondary $(6-10)$ & 48 & 11.9 \\
\hline Intermediate $(11-12)$ & 10 & 2.5 \\
\hline Above 12 & 11 & 2.7 \\
\hline \multicolumn{3}{|l|}{ Marital status } \\
\hline Single & 29 & 7.2 \\
\hline Married & 339 & 84.3 \\
\hline Divorce/separated/widow & 34 & 8.5 \\
\hline \multicolumn{3}{|l|}{ Employment status } \\
\hline Housewives & 366 & 91 \\
\hline Unemployed & 18 & 4.5 \\
\hline $\begin{array}{l}\text { Others (teachers, students, daily wages, Lady } \\
\text { health worker, tailor) }\end{array}$ & 18 & 4.5 \\
\hline $\begin{array}{l}\text { Duration of breast cancer in months Mean } \\
\text { (SD) }\end{array}$ & $28.97(19.93)$ & \\
\hline
\end{tabular}


Depression was present in $279(69.4 \%)$ women and 191 (47.5\%) reported suicidal ideation on item no. 9 (thoughts that you would be better off dead, or of hurting yourself) of PHQ-9. A total of 240 (59.7\%) women were reported to be anxious on GAD-7 (Table 2).

Table 2: Prevalence of depression, anxiety and suicidal ideation $(\mathrm{N}=402)$

\begin{tabular}{|l|c|c|}
\hline Scale & n & $\%$ \\
\hline PHQ-9 & & \\
\hline$\geq 10$ (Presence of depression) & 279 & 69.4 \\
\hline$<10$ (Absence of depression) & 123 & 30.6 \\
\hline Mean (SD) & $11.44(5.21)$ & \\
\hline GAD-7 & & \\
\hline$\geq 10$ (Presence of anxiety) & 240 & 59.7 \\
\hline$<10$ (Absence of anxiety) & 162 & 40.3 \\
\hline Mean (SD) & $9.40(4.49)$ & \\
\hline Both depression ( 10 ) and anxiety $\geq 10)$ & 217 & \\
\hline Suicidal Ideation (Item 9 on PHQ-9) & & 54 \\
\hline$\geq 1$ (Suicidal ideation present) & 191 & \\
\hline 0 (Suicidal Ideation absent) & 211 & \\
\hline
\end{tabular}

Moreover, 217 (54\%) participants had both anxiety and depression (Table 2). Findings also show that majority $(66.2 \%)$ of the women had moderate or moderately severe depression. Similarly, majority of the participants reported moderate anxiety (Table 3). 
Psychological Comorbidity of Breast Cancer ...

Table 3: Distribution of patients across severity categories of depression and anxiety ( $N=402)$

\begin{tabular}{|l|c|c|}
\hline Severity categories & Depression & Anxiety \\
& $\mathbf{N}(\%)$ & N (\%) \\
\hline No-minimal & $63(15.5)$ & $74(18.4)$ \\
\hline Mild & $60(14.9)$ & $88(21.9)$ \\
\hline Moderate & $159(39.6)$ & $240(59.7)$ \\
\hline Moderately severe & $107(26.6)$ & - \\
\hline Severe & $13(3.2)$ & - \\
\hline Total & $402(100)$ & $402(100)$ \\
\hline
\end{tabular}

\section{Discussion}

Findings of this cross sectional study report high rates of anxiety, depression and suicidal ideation in breast cancer patients in Karachi, the largest metropolitan city in Pakistan.

Results are consistent with previous evidence on psychological comorbidity of breast cancer (Jeffery, Russo, Hopkins, \& Burke, 2017). Prevalence of depression in breast cancer patients reported in the current study (69.4\%) are almost double as compared to the global prevalence rate reported by a recent systematic review (32.2\%) (Pilevarzadeh et al., 2019). Previous evidence show that depression is a frequently diagnosed mental health disorder among Pakistani women (GodIl et al., 2017). Majority of women in Pakistan are not financially well-equipped (Tarar \& Pulla, 2014) and are either unemployed or working on daily wages in the informal sector. Similar trends are observed in the current study. This may lead to increased vulnerability in Pakistani women not just for depression, but also for other chronic diseases (Rafique et al., 2018), as well as challenges associated with multimorbidity (Singh et al., 2019). Additional challenges caused by multimorbidity may effect women's quality of life and prevent them from active participation in income generation for the family (DiGiacomo, Green, Rodrigues, Mulligan, \& Davidson, 2015).

Depression is the most common psychiatric risk factor for suicidal ideation and suicide behaviours (WHO, 2014). Along with depression, the current study also reports high prevalence of suicidal ideation (47.5\%) in breast cancer patients. Existing evidence show that approximately 1 out of 10 breast cancer patients reports suicidal ideation one year post-surgery (Kim et al., 2013). Suicidal ideation can be a potential risk of completed 


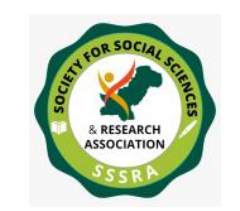

Pak. Journal of Int'L Affairs, Vol 4, Issue 2 (2021) Psychological Comorbidity of Breast Cancer ...

suicide, which is a major public health concern. Every year more than 800,000 people kill themselves by suicide which estimates to 1 suicide after every 40 seconds (WHO, 2014). Most of these suicides occur in low and middle income countries therefore high suicidal ideation in this vulnerable group of breast cancer patients may have serious implications for low resource settings like Pakistan.

The current study also report high rates of anxiety (66.2\%) in breast cancer patients and findings are consistent with prevalence of anxiety (41.9\%) reported by a recent review (Hashemi et al., 2020). Anxiety is usually triggered by worries and fear of recurrence of the disease and death (Ellegaard, Grau, Zachariae, \& Bonde Jensen, 2017).

To authors' knowledge, this study is one of the largest studies from Pakistan to estimate psychological comorbidity of breast cancer in Pakistan. However, the study has certain limitations as well. Structured self-report questionnaires were used to evaluate rates of depression and anxiety and no diagnostic instrument was used to confirm the diagnosis. Moreover, the study included all breast cancer patients regardless of their stage of illness. It would be worth exploring association of psychological comorbidity with stage of illness in future studies. Using a convenient sampling may also warrant caution on generalizability of the findings. Furthermore, some potential variables may act as confounding variables such as stressful life event, premorbid psychiatric history, social support and others which should be explored in details in future studies.

\section{Conclusion}

Findings from this study may have significant implications for patient information, awareness and education, screening for comorbid psychosocial problems. Early detection of and effective culturally appropriate interventions for management of symptoms of depression and anxiety in cancer patients designed to best meet their needs are of huge public health and clinical significance as they will help to improve survival in patients with breast cancer.

\section{Acknowledgement}

We would want to thank all the breast cancer patients for their participation in this research. Authors want to acknowledge Pakistan Institute of Living and Learning for funding this study as part of community development initiative. We also want to acknowledge Prof Nusrat Husain (University of Manchester), Prof Imran B Chaudhry (Ziauddin University and Hospital) and Prof Nasim Chaudhry (PILL) for their regular supervision in design of the project, support in getting permission for data collection and 
reviewing the manuscript. We want to thank Mr Mohsin $\mathrm{H}$ Alvi for reviewing the manuscript. We also want to thank Mrs Zaib Ul Nisa for helping with data entry and $\mathrm{Mr}$ Suleman Shakoor for supporting us in statistical analysis.

\section{Disclaimer}

The abstract or article has not been presented at or published in any conference. The study is part of a $\mathrm{PhD}$ thesis.

\section{Conflict of Interest}

None to declare

\section{Funding Disclosure}

The study was funded by the Pakistan Institute of Living and Learning (PILL) as part of its community development initiative. 


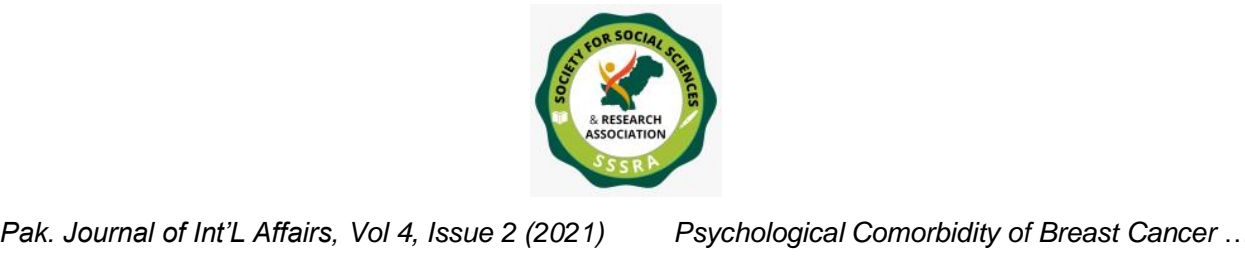

\section{References}

Ahmad, S., Hussain, S., Akhtar, F., \& Shah, F. S. (2018). Urdu translation and validation of PHQ-9, a reliable identification, severity and treatment outcome tool for depression. $J$ Pak Med Assoc, 68(8), 1166-1170.

Ahmad, S., Hussain, S., Shah, F. S., \& Akhtar, F. (2017). Urdu translation and validation of GAD-7: A screening and rating tool for anxiety symptoms in primary health care. $J$ Pak Med Assoc, 67(10), 1536-1540.

Basra, M., Saher, M., Athar, M. M., \& Raza, M. H. J. A. P. J. C. P. (2016). Breast cancer in Pakistan a critical appraisal of the situation regarding female health and where the nation stands. 17, 3035-3041.

DiGiacomo, M., Green, A., Rodrigues, E., Mulligan, K., \& Davidson, P. M. (2015). Developing a gender-based approach to chronic conditions and women's health: a qualitative investigation of community-dwelling women and service provider perspectives. $B M C$ women's Health, 15(1), 1-11.

Ellegaard, M.-B. B., Grau, C., Zachariae, R., \& Bonde Jensen, A. (2017). Fear of cancer recurrence and unmet needs among breast cancer survivors in the first five years. A cross-sectional study. Acta Oncologica, 56(2), 314-320.

GodIl, A., Mallick, M. S. A., Adam, A. M., Haq, A., Khetpal, A., Afzal, R., . . Shahid, N. (2017). Prevalence and severity of depression in a Pakistani population with at least one major chronic disease. Journal of Clinical Diagnostic Research: JCDR, 11(8), OC05.

Hashemi, S.-M., Rafiemanesh, H., Aghamohammadi, T., Badakhsh, M., Amirshahi, M., Sari, M., ... Roudini, K. (2020). Prevalence of anxiety among breast cancer patients: a systematic review and meta-analysis. Breast Cancer, 1-13.

Jeffery, D., Russo, C. A., Hopkins, L., \& Burke, H. B. (2017). Mental health comorbidity costs and healthcare utilization in a nonelderly population with breast cancer. Paper presented at the PSYCHO-ONCOLOGY.

Khan, S., Khan, N. A., Rehman, A. U., Khan, I., Samo, K. A., \& Memon, A. S. (2016). Levels of depression and anxiety post-mastectomy in breast cancer patients at a public sector Hospital in Karachi. Asian Pacific Journal of Cancer Prevention, 17(3), 1337-1340.

Kim, J. M., Jang, J. E., Stewart, R., Kim, S. Y., Kim, S. W., Kang, H. J., . . Yoon, J. S. (2013). Determinants of suicidal ideation in patients with breast cancer. Psycho-oncology, 22(12), 2848-2856.

Kroenke, K., Spitzer, R. L., \& Williams, J. B. (2001). The PHQ-9: validity of a brief depression severity measure. J Gen Intern Med, 16(9), 606-613. doi:10.1046/j.15251497.2001.016009606.x

Löwe, B., Decker, O., Müller, S., Brähler, E., Schellberg, D., Herzog, W., \& Herzberg, P. Y. (2008). Validation and standardization of the Generalized Anxiety Disorder Screener (GAD-7) in the general population. Medical care, 266-274. 


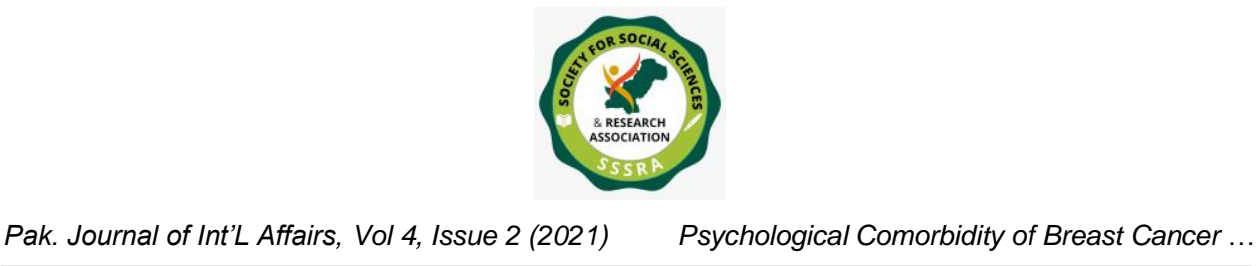

Maroufizadeh, S., Omani-Samani, R., Almasi-Hashiani, A., Amini, P., \& Sepidarkish, M. (2019). The reliability and validity of the Patient Health Questionnaire-9 (PHQ-9) and PHQ-2 in patients with infertility. Reprod Health, 16(1), 137. doi:10.1186/s12978-0190802-X

Menhas, R., \& Umer, S. (2015). Breast Cancer among Pakistani Women. Iranian journal of public health, 586-587.

Panagioti, M., Stokes, J., Esmail, A., Coventry, P., Cheraghi-Sohi, S., Alam, R., \& Bower, P. (2015). Multimorbidity and patient safety incidents in primary care: a systematic review and meta-analysis. PloS one, 10(8).

Pilevarzadeh, M., Amirshahi, M., Afsargharehbagh, R., Rafiemanesh, H., Hashemi, S.-M., \& Balouchi, A. (2019). Global prevalence of depression among breast cancer patients: a systematic review and meta-analysis. Breast cancer research treatment, 1-15.

Rafique, I., Saqib, M. A., Munir, M. A., Qureshi, H., Rizwanullah, K. S., Khan, S. A., \& Fouad, H. (2018). Prevalence of risk factors for noncommunicable diseases in adults: key findings from the Pakistan STEPS survey. East Mediterr Health J, 24(1), 33-41.

Ranganathan, K., Singh, P., Wilkins, E. G., Hamill, J. B., Aliu, O., Newman, L., . . Momoh, A. O. (2018). The Global Macroeconomic Burden of Breast Cancer: Implications for Oncologic and Reconstructive Surgery. Plastic Reconstructive Surgery Global Open, 6(4 Suppl).

Rogers, L. Q., Courneya, K. S., Anton, P. M., Verhulst, S., Vicari, S. K., Robbs, R. S., \& McAuley, E. (2017). Effects of a multicomponent physical activity behavior change intervention on fatigue, anxiety, and depressive symptomatology in breast cancer survivors: randomized trial. Psycho-oncology, 26(11), 1901-1906.

Shang, X., Hodge, A. M., Peng, W., He, M., \& Zhang, L. J. C. (2020). Are Leading Risk Factors for Cancer and Mental Disorders Multimorbidity Shared by These Two Individual Conditions in Community-Dwelling Middle-Aged Adults? , 12(6), 1700.

Singh, K., Patel, S. A., Biswas, S., Shivashankar, R., Kondal, D., Ajay, V. S., . . Kadir, M. M. (2019). Multimorbidity in South Asian adults: prevalence, risk factors and mortality. Journal of Public Health, 41(1), 80-89.

Spitzer, R., Kroenke, K., \& Williams, J. (2006). Generalized anxiety disorder 7-item (GAD-7) scale. Arch. Intern. Med., 166, 1092-1097.

Tarar, M. G., \& Pulla, V. (2014). Patriarchy, gender violence and poverty amongst Pakistani women: A social work inquiry. International Journal of Social Work Human Services Practice, 2(2), 56-63.

WHO, W. H. O. (2014). Preventing suicide: A global imperative.

WHO, W. H. O. (2018). Breast Cancer Statistics. 